\title{
Teaching Creativity: The Case for/Against Genetic Algorithms as a Model of Human Creativity
}

\author{
John L. Dennis ${ }^{*}, 1,2$ and Aldo Stella ${ }^{3,4}$ \\ ${ }^{I}$ Department of Clinical and Experimental Medicine, Psychiatry Section, University of Perugia, Italy \\ ${ }^{2}$ Department of Psychology, Catholic University of Sacred Heart, Milan, Italy \\ ${ }^{3}$ Department of Comparative Cultures, University for Foreigners, Perugia, Italy \\ ${ }^{4}$ Department of Psychology, University "La Sapienza", Rome, Italy
}

\begin{abstract}
In this article we discuss how the creative processes can be modelled by genetic algorithms (GAs). Three fundamental aspects of human creativity need to be considered: 1) the emergence of a novel element;2) the use of that element to achieve a goal; 3) the presence of obstacles imposed by the problem space. GAs have been proposed as a model of these aspects of human creativity. A GA, despite following procedures that are not intelligent is able to find solutions that can be recognised as intelligent. Examining GAs it is possible to prescribe principles that can be useful for human creativity. These principles include the memorisation, storage and retrieval of information as well as the combination and recombination of simple elements within a mechanical procedure of trial and error that formulate the foundation of a cumulative process. GAs can have an important role in the educational process. Education must teach not only rules and schemas as well as the capacity to focus on what information is relevant, but also must teach one to be open to new information and be elastic with it such that that which doesn't immediately seem useful can remain for some time for "trial and error" experimentation that can produce results that are typical of human creativity. Combing these two fundamental aspects of human creativity (rules and elasticity) configures a model of education that can develop a mind that is both rigorous as well as creative.
\end{abstract}

Keywords: Human creativity, generative creativity, genetic algorithms, experiential learning, education, neural networks.

\section{INTRODUCTION}

A number of articles [1-8] describe how genetic algorithms (GAs) can be used as a model for creative thought and how these models can achieve truly novel results. In this paper, our goal is to summarise several thinking style principles, inspired by GAs, which can be useful for young people. These principles will instruct them how to collect, store, retrieve and process information.

When it comes to the creative process, one should consider at least three key factors: 1) The novelty that occurs by virtue of the creative process, 2) functionality that the new product performs in relation to a particular objective, 3 ) and the constraints of problem spaces that limit both the creative process and that suggest solutions that are novel as well as functional [9-11].

The principles of GAs can be useful tools to traverse problem spaces [12]. In fact, by using the mechanical procedure of trial and error, a cumulative process is determined that is configured as a combination and recombination of elements and element sets. Indeed, we could say that every time a search process reaches a solution that is both unexpected and effective, then we are faced with

\footnotetext{
*Address correspondence to this author at the Department of Clinical and Experimental Medicine, Psychiatry Section, S. Maria della Misericordia Hospital, S. Andrea delle Fratte, 06156 Perugia, Italy;

Tel: +39.075.578.4100; Fax: +39.075.573.0537;

E-mail: j.lawrence.dennis@gmail.com
}

a process that could be modelled using GAs. While the process is not intelligent, the solution may be intelligent when it is judged (by a human) to be both innovative and functional.

To achieve the goal of defining the benefits that may result from an education in creative thinking using principles configured by GAs, we will first discuss the theoretical and historical foundations of GAs and then we will move our discussion to the basic characteristics of GAs. From here we will then discuss how GAs can be used in the creative process and finally we will discuss the relationship between GAs and the educational process, trying to show how an education process should combine rules and schemas with flexibility and openness to novelty. The education process should, in short, teach students how to discern moments when it is advisable to filter incoming information, to avoid unnecessary high consumption of cognitive resources and when it is advisable to take in a greater amount of information because this greater amount of information will be used to construct those building blocks that when properly combined will produce a new edifice of knowledge.

This present paper describes briefly the history of GAs and that their history is derived, on the one hand, from neural networks, and on the other hand, from the Darwinian model of evolution. We then discuss the relationship between Campbell's BVSR model, Universal Darwinianism and Hull's GAs. After describing the structure of the GAs and an abstract example of the program, we trace the characteristics 
of GAs as a possible model of human creativity and clarify the limits of GAs as well. We conclude by discussing the usefulness of GAs for education in general as well as the education of creativity.

\section{FROM NEURAL NETWORKS TO GAs}

Modern computing began with sequential operations specified formally in programming code following the socalled von Neumann architecture. Artificial intelligence described in the classical cognitive or symbolic models is a program that elaborates information in accordance with rules where information is reflected in the form of representations. These representations are handled in accordance with set procedures that are not random and the program itself is the set of rules that instruct a priori the calculation procedure [13]. Neural network or sub-symbolic models, which can be seen as biologically inspired connectionist models [14-16] were designed to solve complex problems, function according to a predetermined series of steps that dictate each transaction according to an a priori program that resolves problems on the basis of operational units called "neurons" (terminology inspired by biology) [17]. The units are interconnected with each other and are distinguishable between orders: the units of input, output and the intermediate units. The interesting thing is that the interconnections between the units that make up the network can change constantly based on feedback on the success (or failure) of the network in dealing with a problem. The key feature of neural networks is that they solve problems without a priori knowledge of the answer, the steps needed to achieve it and a clear understanding by the programmer how the problem can be solved in some form of logical procedure. Neural networks, generate solutions by a mechanism of "trial and error." This generation is progressive, meaning that it gradually and spontaneously approaches the "best" solution that configures operational success on the basis of the forms that did not get it, but are rewarded by the fact that they approach it gradually. The architecture of the trained network (i.e., programmed to obtain a particular result) is the result of the history of the network itself, or of its experience. This approach is not a "logical" approach to computation, but "empirical," in that it is based on the network's experience [17].

\section{THE CONNECTION: HULL'S GAS, CAMPBELL'S BVSR AND UNIVERSAL DARWINISM}

Genetic algorithms are class of search methods especially suited for solving complex problems [12, 18, 19]. As stated earlier, they transpose the notion of evolution systems by imitating natural evolution with computers. Genetic algorithms were initially introduced by Holland [20] based, in part, on Campbell's [2] "blind variation and selectiveretention" (BVSR).

Campbell [2] considered the process of arriving at a thought as a selection among the possible methods for varying that thought in order to generate a subsequent thought and then by repeating this process a new thought is produced. In this way Campbell envisioned thought as being "blind" - in that it is produced without any objective/goal. Campbell also stressed the "selective-retention" of thought in that there must be a memory for information.
Universal Darwinism is a core idea that makes the application of GAs to human creativity possible. Universal Darwinism is the idea that Darwinian evolution is not limited to explain the evolution of the physical structures of organic life but it could be used to explain other substrates [21] from the evolution of antibodies by immune systems [22] and the selective pruning of neural circuitry in neural systems [23] as well the evolution of thought [24] and culture [25].

\section{GENETIC ALGORITHMS AS A MODEL OF DAR- WINIAN EVOLUTIONARY}

Genetic algorithms represent the evolution of neural networks in virtual environments [20]. They mimic biology, with regard to replication, mutation and genetic recombination. A population of artificial networks representing virtual organisms that change gradually under the selection and reproduction of those networks is based on successful behaviour in a simulated environment. Random changes in the structure and function of the connectivity of the "first" forms can persist or not in forms descended on the basis of their operational success. This model helps to explain how the system will be progressively updated under the empirical success or failure of product behaviour [26]. The consequence is that the configurations of the system that best exemplify the success evolve, where they tend to fall less useful configurations. Natural selection is, therefore, the main force and the random genetic variations that are operating on natural selection [19].

In the development of evolutionary systems, the process takes the following steps: 1) Initialisation: This function creates a population of random solutions that are called "chromosomes". 2) Fitness Test: This function assigns to each chromosome of the population an adaptive value (fitness) in relation to its ability to solve the problem. 3) Reproduction: The chromosomes with higher fitness are more likely to reproduce, creating new solutions. 4) Next Generation: If the optimal solution has been reached, the process stops, otherwise the process continues by creating a new generation [20].

The GAs can be described abstractly in software language as following this procedure:

\section{Creates initial population}

Produces a first selection of the population

Repeat

\section{Creates new population}

Chooses individuals

Couples them

Generates offspring

Induces mutations in selected offspring

Chooses and retains individuals

\section{Terminate}

To clarify the initial population is created randomly and then this population is evaluated using the fitness function (scaled as rank-based, proportional, truncated, etc.) or the conformity of the individuals of the population in relation to 
the goal. This initial population is used to create a new population where individuals are selected for mating according to various selection mechanisms (e.g., fitness proportional, tournament, ranked, uniform, roulette, etc.) Offsprings are generated by applying genetic operators, i.e., crossovers mechanisms, (e.g., heuristic, scattered, singlepoint, etc.) to the parents who combine their genetic code when mating. Mutations are induced in the code of a select few children, where the mutations are generally selected on the basis of characteristics of the specific problem that needs to be solved, i.e., the objective to be achieved. These types of mutations are often referred to as adaptive feasible mutations, but other mutations can be programmed, for example, Gaussian mutations. The operations indicated repeat until an offspring occurs (solution) that exhibits values that conform to the objective that has been previously established.

\section{USEFUL FEATURES OF GAS AS MODELS OF HUMAN CREATIVITY}

Solving a problem with GAs starts with designing a proper representation, fitness measure and termination criterion. The mechanics of this process points to significant useful features for the modelling of human creativity [27]. A core feature of GAs is cumulative progress, whereby each new instance (idea, behaviour, etc.) is based on past ones. The evaluation process of GAs, whereby past instances to the development of new ones is directly and positively related to the utility of those past instances and runs parallel to cognitive mechanisms in human problem solving.

A GA's capacity for hierarchical structure enables the algorithm to discover useful combinations of previously discovered building blocks, which then become new building blocks. This recursive process can lead to instances that have a hierarchical structure. Recombination, such that new instances are obtained as hybrids of past instances, thus (often) taking some useful features from past instances. The use of building blocks, or the combinations of features that increase utility (fitness) relative to the population average will become more prevalent. Once they spread through a significant proportion of the population, these feature clusters play essentially the same role as individual features, and are termed building blocks. This is a significant advantage over standard learning algorithms, which only increase the prevalence of advantageous individual features. Together, recombination and building blocks allow for the modelling of the discovery process via novel combinations of previously learned components [24].

Another feature that is analogical to human creativity is that GAs are programmed with parallelism across instances, whereby multiple candidates can be considered simultaneously, and multiple past instances can be combined to create a new instance (this capacity can be limited to simulate the limits on human working memory). Genetic algorithms are also programmed with parallelism across building blocks whereby the algorithm effectively performs simultaneous searches across the power set of all features. That is, the various instances in the population collectively implement separate and simultaneous searches through the feature space associated with each dimension as well as through the product spaces defined by all combinations of dimensions [28].

Novelty is effectively programmed into GAs because it effectively has an unbounded state space. The nature of the state space, as the set of binary or n-ary strings of a given length, leads to an exponential explosion of possibilities that is effectively infinite (in both the context of computation and the context of human ideas or behaviours). Genetic algorithms use of building blocks and its capacity for hierarchical structure combined with unbounded state space enables the potential for perpetual novelty [29]. This effectively unlimited trajectory of innovation may be seen as the true hallmark of human creativity. Furthermore, novelty itself (i.e., variation from the present norm) can be directly selected for by using an evaluation function that takes the present makeup of the population as input [30].

Implicit in the idea of building blocks is the idea that certain sets of features work far better in conjunction than individually (whole $>$ parts). It is precisely this fact of nature that makes the combinatorial explosion in the search space so important (making the search an NP-hard problem) and thereby makes creativity so crucial to success in many realworld scenarios. The parallelism across building blocks and the discovery of building blocks and the capacity for hierarchical structure of building blocks are processes by which the GAs essentially seeks out, recognizes and exploits these non-linearity, and incorporates them into its representational language [30].

One essential challenge for GAs is the method for the unitisation of building blocks. With GAs the recombination of two (or more) parent instances is done essentially at random. A crossover point is selected and the substrings following that point are exchanged [31]. Recombination in human cognition does not appear to be as arbitrary; rather, acknowledged building blocks are usually kept intact. Thus a GAs model of creativity may need to include a mechanism whereby crossover points are learned so as to selectively reduce the probability of breaking up building blocks. One simple possibility is to have each instance include "bond strengths" associated with the links between all consecutive pairs of genes. The bond strengths would themselves be treated as genes (perhaps appended to the end of the string) and would thus be subject to selection in the same manner as the primary genes.

\section{LIMITATIONS OF GAs}

The questions we ask are as follows: Can GAs be used to model human creativity? What relevance do they have for problem solving in general and the learning process at large? It seems to us that if we speak of a tacit or implicit learning, then genetic algorithms may help explain some part of the learning process [32]. If, in contrast, when we speak of conscious learning, and conscious problem solving, which implies reflective and creative thinking, then genetic algorithms may be less appropriate for explaining the learning process [5]. The GAs describes, in short, a form of learning that is automatic, where information is randomly and automatically selected, retained, stored and retrieved, whereas in the case of conscious learning information is selected by choice and with effort it is selected, retained, stored and retrieved. 
A fundamental point of departure for GAs as a model of creative thought processes is the relationship between inductive and deductive processes. Conscious thought, in short, is not only reproductive (as GAs are) but it is productive in that it produces new knowledge. GAs seem to resolve this problem by uniting the inductive as well as the deductive processes. GAs are deductive because they are procedural calculation, but a GAs adds something in that the results increase the amount of information that is present in the premises - which is exactly what happens in inductive processes [20]. It is therefore possible to describe GAs as a crossroad between inductive and deductive processes.

There is perhaps an incompatibility between the randomness of evolutionary mechanisms which are the foundation of GAs and the foundations of human conscious thought [33]. If the production of new knowledge needs to follow both inductive and deductive processes, then the Popperian model of "conjectures and refutations", which is an intelligent process [34] is the appropriate model. But GAs are not based on the Popperian model, rather GAs use trial and error mechanisms which do not provide for guessing, hypothesis testing, or thought in the production of new knowledge [8].

Yet another problem for GAs is what Perkin's describes as unreasonable problems [35]. These type of problems are considered unreasonable because finding the solution to these problems often needs insight from an outside source whereas in reasonable problems where the solution is discovered by working through a step-by-step process. While GAs can produce results that are "unexpected", but the model is essentially deterministic, while human insight produces unexpected results that are "in-deterministic" in that they can only be described post-hoc as an interaction between the context and the problem [10].

If there is an incompatibility between the randomness of the underlying evolutionary mechanisms of GAs and the conscious goal-orientedness of the underlying mechanisms of human thought then the correct role of conscious thought is in the programming as well as the evaluation of the GAs output. Thus, conscious thought is valuable in the world of GAs for the conscious understanding of the problem, the process of finding the problem, the examples that will be stored in the GAs, the setting of the problems goal (the fitness function), the evaluation of the solution of the problem, and, perhaps most importantly the programming of the GAs. Indeed, given the fact that the program itself is blind, the role of the programmer is fundamental to establish and define a priori the problem space including its initial state, and set the a posteriori limits that consent to define the fitness of the problem space.

\section{EDUCATION AND GENETIC ALGORITHMS}

How then can GAs be used in an educational setting? The principles of the GAs model can be used to teach the student to think in a non-schematic way, such that a multitude of information is taken into account and this information remains activated for later elaboration processes and at a later moment the student can individuate the elements that can be useful for solving the problem. Practically, this means that the learner should not discard possible solutions as new iterations are developed. In both
Hull's GAs as well as Campbell's BVSR models both require that in successive iterations of problem solving possible solutions are present and processed in parallel rather than serially. Serial models perhaps best describe human problem solving in that solutions are considered successively. This difference leaves significant room for GAs to help in problem solving in that if human creativity is the actualisation via exposure to various contexts it seems relevant to keep possible solutions available in parallel for future consideration.

GAs can also be used to describe how this non-filtering of information should proceed, i.e., by trial and error. This type of learning/thinking is one that is related to operant conditioning models [6] in that the behaviour of creativity can be such that formulating the solution of a problem as a playful game can help one significantly in solving the problem. In addition, following the principles of GAs, learners should be advised to store a large number of examples and then use those stored examples at the right moment to begin the creative process (i.e., the combination and recombination stage).

Of course, teaching the learner how to proceed as if they were, at least in part, processing information mentally like a GAs as it has been described previously is one way that GAs can be used in the education process. Another way that GAs can be used in the education process is to teach the learner how to use GAs in the form of software that can be individuated to their needs. Therefore, when the learner cannot find a creative solution to a problem, the learner can use a GAs to look for a possible solution.

An education that integrates processes that are inspired by GAs as well the goal orientated feature of human thought that move it towards objectives. This way one creates an intelligence that is creative in that it "trusts" combinations of information and processes of the elaboration of information that are based on chance, but constantly evaluate those efforts with the conscious goal of the problem in mind.

\section{ACKNOWLEDGEMENTS}

This research was supported by Cassa di Risparmio di Perugia Fellowship to John L. Dennis and a University for Foreigners Research Grant to Aldo Stella. John L. Dennis would like to thank Matt Jones and Brad Love for their helpful discussions and comments on the ideas (when they were in the incubation period so many years ago) that became the foundation of this current article. John L. Dennis would also like to thank Roberto Quartesan for giving him the intellectual space to write this article.

\section{CONFLICT OF INTEREST}

None declared.

\section{REFERENCES}

[1] Bradshaw GF, Langley PW, Simon HA. Studying scientific discovery by computer simulation. Science 1983; 222(4627): 9715.

[2] Campbell DT. Blind variation and selective retention in creative thought as in other knowledge processes. Psychol Rev 1960; 67: 380-400.

[3] Cziko G. From blind to creative: In defense of Donald Campbell's selectionist theory of human creativity. J Creat Behav 1998; 32: 192-208. 
[4] Langley P, Simon HA, Bradshaw GL, Zytkow JM. Scientific discovery: computational explorations of the creative processes. MA: MIT Press 1987.

[5] Perkins D. Creativity: Beyond the Darwinian paradigm. In: Boden MA, Ed. Dimensions of creativity. Cambridge, MA: MIT Press 1994.

[6] Simonton DK. Creativity as cognitive selection: The blindvariation and selective-retention model. Behav Brain Sci 2001; 24(3): 554-6.

[7] Smith SM, Ward TB. The evolution of creativity. In: Rosen D.H, Gardner R, Luebbert M, Eds. Evolution of the Psyche. Westport, CA: Greenwood Publishing Group 1999.

[8] Sternberg RJ. Cognitive mechanisms in human creativity: Is variation blind or sighted? J Creat Behav 1998; 32: 159-76.

[9] Smith SM, Ward TB, Finke RA, Eds. The creative cognition approach. Cambridge: MIT Press 1995.

[10] Weisberg R. Creativity: genius and other myths. New York: Freeman 1986.

[11] Perkins D. The minds best work. Cambridge, Massachusetts: Harvard University Press 1981.

[12] Holland JH. Adaptation in natural and artificial systems. Ann Arbor, MI: The University of Michigan Press 1975.

[13] Turing AM. Computer machinery and intelligence. Mind 1950; 49: 433-60.

[14] Rumelhart DE, Smolensky P, McClelland JL, Hinton GE. Schemata and sequential thought processes in PDP models. In: McClelland JL, Rumelhart DE, Eds. Parallel Distributed Processing: Explorations in the microstructure of cognition. Cambridge, MA: MIT Press 1986.

[15] Burgess N, Hitch GJ. Memory for serial order: A network model of the phonological loop and its timing. Psychol Rev 1999; 106(3): 551-8.

[16] Gluck MA, Bower GH. From conditioning to category learning: An adaptive network model. J Exp Psychol Gen 1988; 117(3): 227-47.

[17] Anderson JA. An Introduction to Neural Networks. Cambridge, MA: MIT Press 1995.

[18] Bentley P, Ed. Evolutionary design by computers. San Francisco, CA: Morgan Kaufmann Publishers 1999.
[19] Goldberg DE. Genetic algorithms in search, optimization, and machine learning. Addison-Wesley 1989.

[20] Holland JH. Genetic algorithms. Sci Am 1992; 267: 66-72.

[21] Dennett DC. Darwin's Dangerous Idea. New York: Simon and Schuster 1995.

[22] Burnet FM. The clonal selection theory of acquired immunity. London, UK: Cambridge University Press 1959.

[23] Edelman GM. Neural Darwiniasm: The theory of neuronal group selection. New York: Basic Books 1987.

[24] Simonton DK. Creativity as blind variation and selective retention: Is the creative process Darwinian? Psychol Inq 1999; 10: 309-28.

[25] Boyd R, Richerson P. Built for speed, not for comfort: Darwinian theory and human culture. Hist Philos Life Sci 2001; 23: 423-63.

[26] Hull DL, Langman RE, Glenn SS. A general account of selection: Biology, immunology, and behavior. Behav Brain Sci 2001; 24: 511-73.

[27] Koza JR. Genetic programming II: Automatic discovery of reusable programs. Cambridge, MA: MIT Press 1994.

[28] Haupt RL, Ellen HS. Practical Genetic Algorithms. Hoboken, NJ: John Wiley \& Sons, Inc. 2004.

[29] Cziko G. Without miracles: universal selection theory and the second Darwinian revolution. Cambridge, MA: MIT Press 1995.

[30] Goldberg DE. The design of innovation: lessons from and for competant genetic algorithms. Dordrecht, NL: Kluwer Academic Publishers Group 2002.

[31] Holland JH. Building blocks, cohort genetic algorithms, and hyperplane-defined functions. Evol Comput 2000; 8(4): 373-91.

[32] Grefenstette JJ. Conditions for implicit parallelism. In: Rawlins GJE, Ed. Foundations of genetic algorithms. San Mateo, CA: Morgan Kaufmann Publishers, Inc. 1991.

[33] Perkins D. In the country of the blind: An appreciation of Dondald Campbell's vision of creative thought. J Creat Behav 1998; 32(3): 177-91.

[34] Popper K. Objective knowledge: An evolutionary approach. UK: Oxford University Press 1979.

[35] Perkins D. Archimede bathtub: The art and logic of breakthrough thinking. New York: W. Norton and Company 2000.

(C) Dennis and Stella; Licensee Bentham Open.

This is an open access article licensed under the terms of the Creative Commons Attribution Non-Commercial License (http://creativecommons.org/licenses/by-nc/ 3.0/) which permits unrestricted, non-commercial use, distribution and reproduction in any medium, provided the work is properly cited. 